\title{
Understanding human factors in rail engineering: re-analysis of detailed, qualitative data on functions and risks
}

\author{
Brendan Ryan ${ }^{\mathrm{a}, *}$, John R. Wilson ${ }^{\mathrm{ab}}$, Alex Schock ${ }^{\mathrm{b}}$ \\ ${ }^{a}$ Human Factors Research Group, University of Nottingham, Nottingham, UK. NG7 2RD \\ ${ }^{\mathrm{b}}$ Network Rail, Ergonomics National Specialist Team, Melton Street, London, UK
}

\begin{abstract}
The paper reports on the review and re-analysis of information that has been collected in earlier field studies on the functions and associated risks in rail engineering and maintenance. Two methods of Cognitive Work Analysis have been adapted and used to identify and represent important components of the rail engineering system and the situations in which activities occur. Additional classification exercises have been used to determine issues of strategic importance to the organisation, related to the functions and human factors risks in performing these functions. The effectiveness of the methods in this industrial context has been evaluated. Conclusions are drawn on how this type of approach can be used to produce relevant findings on the following: What the organisation knows about roles, functions and descriptions of tasks that are relevant for engineering and maintenance work; (2) the HF risks for today's (and unless things change), tomorrow's railway; (3) how this knowledge can help in determining organisational priorities for future work.
\end{abstract}

Keywords: Rail engineering and maintenance, Function and Activity Analysis, Qualitative Analysis

\section{Introduction}

Rail engineering work (maintenance and renewal of track and infrastructure) needs to be carried out with minimal disruption to normal train operations. This is becoming more difficult in rail networks in which demand for rail travel is increasing, resulting in greater numbers of train paths and fewer opportunities for access for engineering work. In the UK, a number of recent programmes of work have considered the types of process and procedural change that would be needed to speed up access to the track for engineering work, whilst maintaining or improving upon existing levels of safety.

The University of Nottingham, working closely with Network Rail (the infrastructure owner), has provided human factors support to a number of these projects. A range of operational and project staff have been involved, along with specialist support (e.g. for safety risk analysis). The work has included the collection of detailed information on what needs to be achieved in terms of rail engineering (the functions of rail engineering and the activities needed for these). This type of information has been used as a foundation for identification and analysis of the potential risks, considering both current and potential future systems for rail engineering [7].

This paper builds on this earlier work, reporting on a review and re-analysis of the detailed, qualitative information on the functions in rail engineering and the risks associated with these functions. This review and re-analysis has been carried out with a view to answering the following questions: (1) What does the organisation know about roles, functions and descriptions of tasks relevant for engineering and maintenance work (2) What are the HF risks for today's (and unless things change), tomorrow's railway. (3) How does this knowledge help in determining organisational priorities for future work?

\footnotetext{
* Corresponding author. E-mail: brendan.ryan@nottingham.ac.uk
} 


\section{Method}

The research reported in this paper uses two modified stages from Cognitive Work Analysis (CWA) [4] and additional classification exercises as part of a re-analysis of information which has been collected during a series of industry projects to examine and improve the efficiency of processes in rail engineering. Outputs from previous phases of work on the functions and risks in rail engineering and maintenance have been identified, summarised and represented in a new format for the re-analysis in this current piece of work. Firstly, a web based tool, developed previously within Network Rail, has been used to produce a hierarchical representation for rail engineering. This part of the analysis has been used as a means of representing the most important contents from lengthy and detailed qualitative outputs from previous work. It has also been used as a method of exploring and representing links between different components within the system. Secondly, a matrix, inspired by the Contextual Activity Template (CAT), has been used to examine the locations and situations in which activities (within functions) are typically or could be performed. Additional qualitative analyses and classification exercises have focused on two areas. The first has involved clarification of the main factors affecting the performance across the range of functions in rail engineering, through review and additional classification of outputs from previous analyses. The second area of focus has been on collation and interpretation of the risks associated with the performance of the functions, to develop understanding of issues of strategic importance to the organisation.

\section{Results}

\subsection{The hierarchical representation for rail engineering}

\subsubsection{Construction of the hierarchy for representation of rail engineering}

In earlier analyses, five functions for rail engineering have been identified (i.e. planning, access / handover of the line, isolation, delivery of the work, return of the line). In work with Subject Matter Experts (SMEs), more than 20 pages of detailed qualitative information were produced in the analysis of these functions. The method of analysis used five criteria to assess different activities within the func- tions (information needed by staff, tools / equipment / machinery, organisational requirements, difficulties, constraints) and considered issues associated with different roles within these functions, such as the PICOP, ES, COSS and other field based roles (e.g. competent person and handsignaller).

In an effort to represent as much of this type of information as possible in a readily accessible format, a hierarchical representation for rail engineering has been produced in the current piece of work. Figure 1 shows an extract from the representation (similar to work domain maps). Network Rail have used these types of representations previously for analyses of signalling, train control and train driving activities, so the approach is familiar to some experts in parts of the organisation. For this reason the representation in the current work has been constructed using the headings which are used within the organisation (i.e. purpose, functions, tasks, activities, tools) rather than those proposed by others (e.g. Naikar et al, 2006 [4]) . Minimal changes were introduced at the highest levels of the hierarchical structure (i.e. retaining as many of the entries in the "purpose" and "functions" categories as possible from other analyses).

The representation helps to identify links between different components in the system, helping to visualise the important relationships between the components. For example, the extract in Figure 1 shows links associated with the activity for "checking standard documents", including the upward and downward relationships through the hierarchy. A wide range of roles are involved (not shown in full in this extract).

\subsubsection{Initial evaluation of the hierarchical representation for rail engineering}

The approach that has been used is not traditional work domain analysis (or abstraction hierarchy), but uses some of the principles of the CWA methods to represent what has been obtained in previous analyses of functions and activities. The representation has been developed to stay consistent with other analyses in the client organisation. The work is still in development. It will be necessary to establish through discussion with the client, in what way this information can be valuable to the organisation (i.e. is an easily accessible record of functions, tasks, activities and other components useful to the organisation?). In the meantime, some preliminary observations on the representation are included. It is clear that it has the potential to display a lot of information 


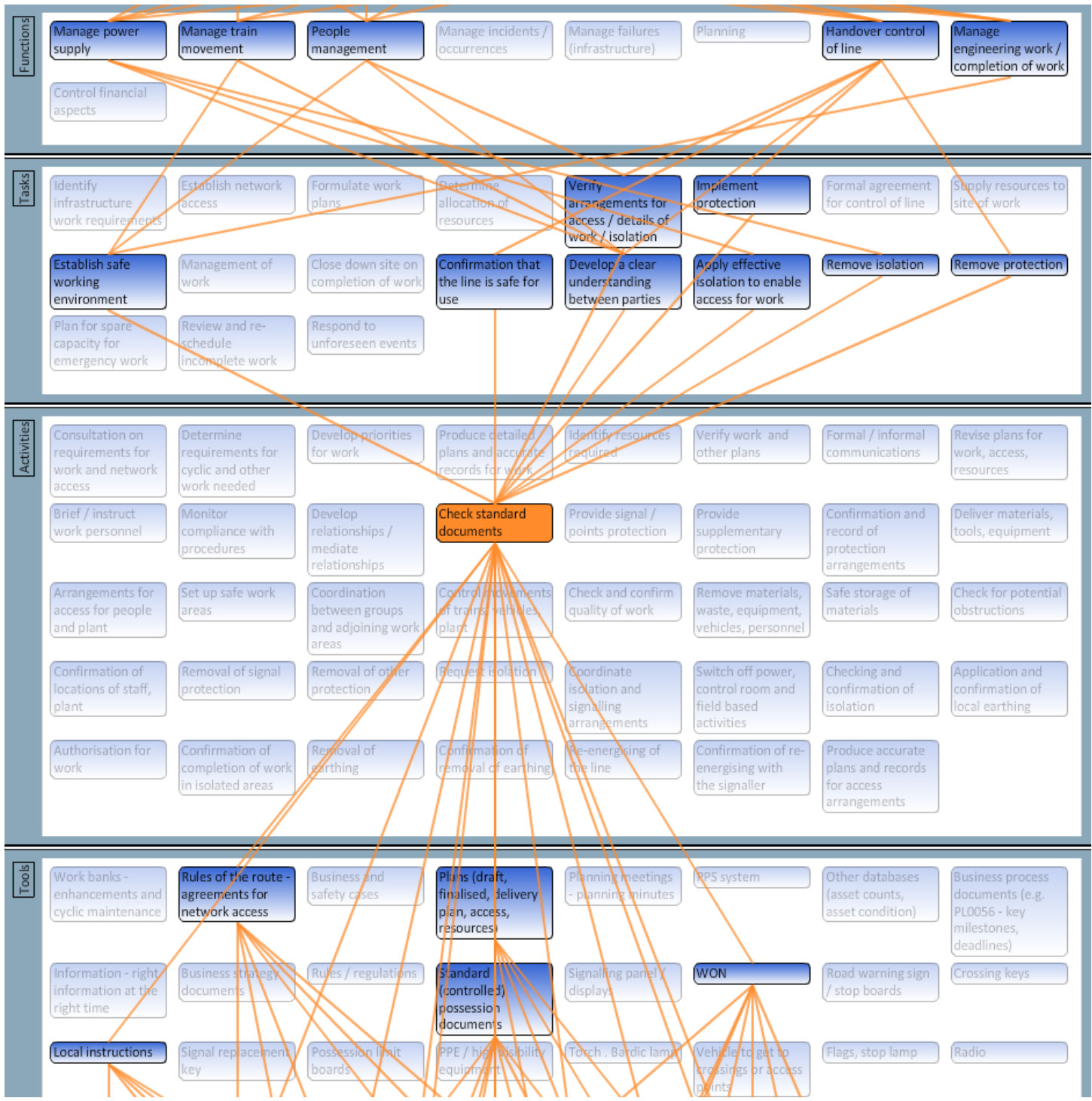

Figure 1 Extract from the hierarchical representation

on a single page of paper. It also has the potential to provide a template for organising a lot of underlying detail. For example, cells in the representation could be linked to a database or knowledge management system, so that a user can find out relevant information related to a task, activity or relevant tools. A considerable amount of detail has already been collected in relation to tasks and activities in earlier parts of the work, though the structuring of this underlying information still needs consideration.

The representation can be used to test and explore relationships between different system components. It is ambitious in its attempt to include activities which are carried out by all engineering related roles and it might be considered to be too complicated in some circumstances (e.g. when showing the high frequencies of linkages from higher levels of the hierarchy). However, this broad view of the system is important in understanding of complexity and inter-relationships. The representation can be used to help to raise questions about functions and other levels in the hierarchy, to build new lines of enquiry (e.g. What activities are important in responding to unforeseen events?). It can also be used in a formative way (e.g. when investigating changes that could occur at activity level, what changes at higher and lower levels would be likely?). The representation 


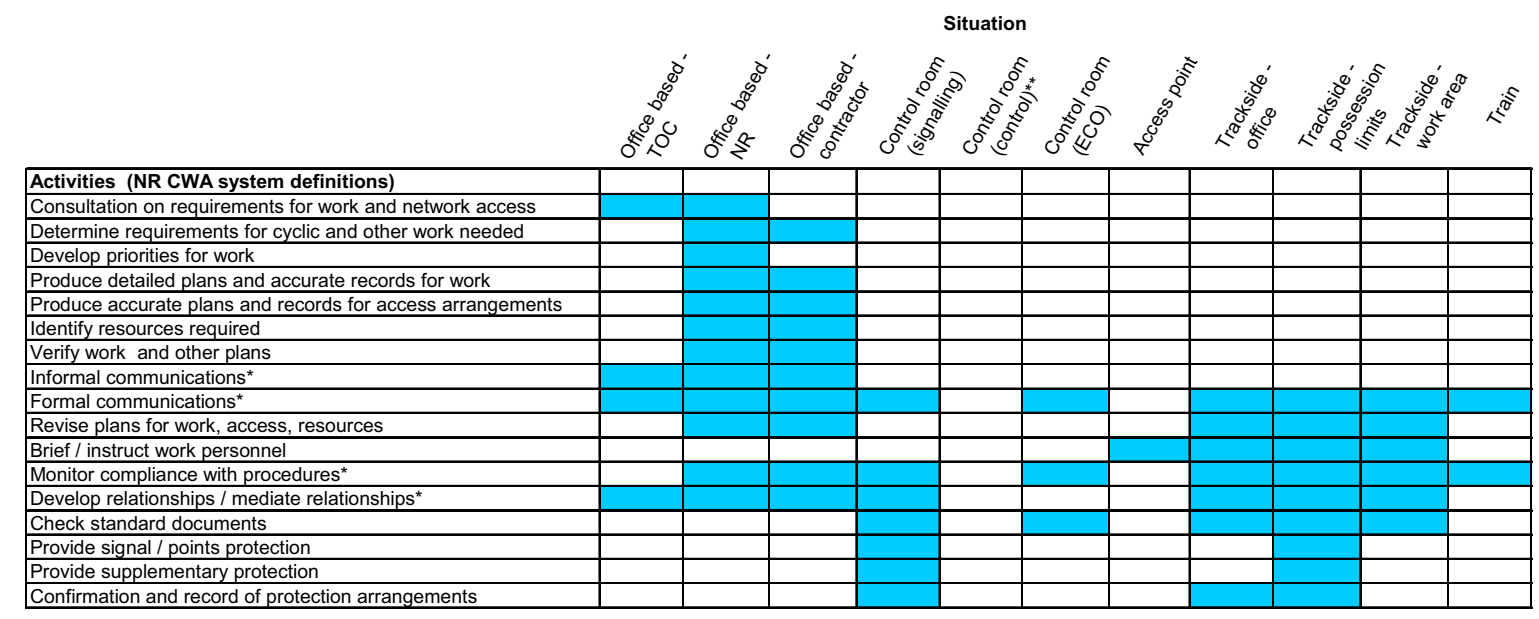

Figure 2 Extract from the matrix

can also be used as an aid to explore things that are uncertain currently, or where there may be gaps in understanding. As an example, the earlier analysis of functions identified 5 main functions in rail engineering. In conducting this re-analysis, these functions have been considered to be at different levels within the hierarchy. The functions for "planning", "access" and "management of the work" have been represented at the "functions" level in the hierarchy (the second level in this representation), whereas "isolation" has been located at a lower level. "Return of the line" has been represented as a part of another function ("handover control of the line"). This is a useful example of how the methodology can enhance understanding of the relationships between system components.

\subsection{The matrix based approach for analysis of activities in rail engineering}

\subsubsection{Construction of the matrix}

The "activities" from the hierarchical representation were used to produce a matrix showing how activities are conducted in different situations or locations (e.g. office, control room and field based locations of activities). Figure 2 shows an extract from the matrix. The cells that are highlighted in the matrix show the situations in which activities occur typically (e.g. checking of standard documents is relevant within control rooms and in a range of track based locations).

\subsubsection{Initial evaluation of the matrix based approach}

This part of the analysis has helped to discriminate the situations in which activities are, or are not, carried out currently. However, there is need for some development in the analysis. The extract from the matrix in Figure 2 does not include any activities within the remit of operational control. This might indicate that Operational Controllers are not involved in these activities, or that more investigation is needed of this role in this rail engineering context. In much the same way that the hierarchical representation can be used to prompt questions about the understanding of the system, the matrix based analysis can also be used as a means of understanding the rail engineering system in greater depth. For example, more work is needed to verify the range of functions associated with the isolation of the different $\mathrm{AC}$ and DC electrical systems and to collect additional information on the constraints on workers and the factors affecting performance of their work. The preliminary matrix could therefore be used as a tool to structure discussions with SMEs about the processes and factors affecting isolations (i.e. focusing on relevant activities, in the situations in which they are known to occur).

This part of the analysis can also be used in a formative way, to understand the implications of future system changes (i.e. to understand what future system changes might look like and what changes in hardware, technology, processes would be needed). Changes might be in relation to the locations in which activities occur (e.g. moving 
Table 1

An extract of the analysis of human factors affecting the function of access / handover of the line

\begin{tabular}{|c|c|}
\hline Explanation of human factors affecting functions & Coding for key human factors \\
\hline $\begin{array}{l}\text { Published arrangements must be fit for purpose } \\
\text { No conflicts with other possessions, regardless of geographical location } \\
\text { No conflicting train movements } \\
\text { Potential for errors in recording details of confirmation, errors in recall of who has called } \\
\text { Potential to act before receipt of confirmation }\end{array}$ & Quality of records / record keeping \\
\hline $\begin{array}{l}\text { Need for sufficient knowledge and experience } \\
\text { Knowledge of key things to check (e.g. if there are implications for adjoining signal boxes) } \\
\text { Knowledge of local infrastructure and site, including safe access points, crossings / crossing } \\
\text { types } \\
\text { Outline knowledge of work that is proposed } \\
\text { Knowledge of rail movements (e.g. towards crossings, possession limits) } \\
\text { Potential impacts of contract, sub-contract staff (e.g. lack of local knowledge, fewer opportuni- } \\
\text { ties for pre-site meetings, control of rostering and working time issues) }\end{array}$ & Level of expertise / experience \\
\hline $\begin{array}{l}\text { Understanding of potential points of conflicts and contingencies (e.g. likely impacts of bank } \\
\text { holidays or major events, late train running, unsuitable access points such as beyond worksite } \\
\text { limits) } \\
\text { Ability to identify and respond to errors in documentation (wrong limits /clashes between } \\
\text { work sites, wrong train paths, incorrect staffing and resources, non-published work) }\end{array}$ & Ability to respond effectively \\
\hline
\end{tabular}

Table 2

Summary of factors affecting the range of functions of rail engineering

\begin{tabular}{|c|c|}
\hline Higher level classification & Key factors affecting performance \\
\hline External influences & $\begin{array}{l}\text { Pressure from stakeholders } \\
\text { External factors (e.g. public) } \\
\text { Time available } \\
\text { Other work / possessions }\end{array}$ \\
\hline $\begin{array}{l}\text { Physical constraints - layout / location } \\
\text { Environmental constraints - including visual cues }\end{array}$ & $\begin{array}{l}\text { Location of work in relation to other infrastructure } \\
\text { Poor weather / visibility } \\
\text { Time of day / visibility }\end{array}$ \\
\hline System variability & $\begin{array}{l}\text { Uncertainty, unpredictability } \\
\text { Change - late changes } \\
\text { Ability to respond effectively }\end{array}$ \\
\hline Organisational & $\begin{array}{l}\text { Degree of standardisation } \\
\text { Quality of processes } \\
\text { Staffing availability } \\
\text { Lengths of shifts, vigilance, fatigue }\end{array}$ \\
\hline Inter-organisational & \\
\hline Coordination / interaction/ teamwork & $\begin{array}{l}\text { Relationships with others / trust } \\
\text { Coordination between workers or groups }\end{array}$ \\
\hline Communication / information flow & $\begin{array}{l}\text { Feedback on performance } \\
\text { Formal and informal communications } \\
\text { Clarity of instructions / communications }\end{array}$ \\
\hline $\begin{array}{l}\text { Individual } \\
\text { Knowledge / expertise }\end{array}$ & $\begin{array}{l}\text { Level of expertise / experience } \\
\text { Knowledge of local infrastructure and train movements }\end{array}$ \\
\hline Understanding of information sources & $\begin{array}{l}\text { Understanding of relevant information sources } \\
\text { Quality of records / record keeping }\end{array}$ \\
\hline
\end{tabular}


activities to a control room rather than trackside), or linked to inclusion of new activities (e.g. change to use of more train based inspection and maintenance). This type of analysis therefore helps to pinpoint where more detailed analyses are needed, including review of existing data sources and collection of additional information, to understand the full implications of changes within the system.

\subsection{Additional analysis of functions, in particular the factors affecting performance of functions}

As explained above, work in an earlier phase focused on the identification, description and analysis of five main functions in rail engineering. Some revisions to this analysis have been carried out as part of the current review. A series of tables have been produced to collate and re-analyse information that was available on the functions. Factors affecting performance of each of the functions have been identified and an example of outputs for the analysis of the function of access / handover of the line is given in Table 1.

A summary of the range of factors affecting performance, across all of the five functions is given in Table 2 .

A relatively small number of factors have been identified that affect the different functions and these could be of strategic and tactical importance to the organisation and help with prioritisation of future work programmes (e.g. whether interventions should be targeted at the level of the individual or the organisation).

In future work, it might be possible to link these factors that can affect performance to the relevant activity / situation combinations that are illustrated in the matrix in Figure 2, giving more depth to the understanding of circumstances (which activities and whether these are relevant to the trackside or in control rooms) in which performance can be inhibited. As an example, it might be possible to pick two or three of the most common "factors" (e.g. coordination) and consider these in more detailed analysis by highlighting where each of these are important on the matrix. Other factors affecting performance, such as "information flow", could be examined using stages of cognitive work analysis (e.g. the social interactions stage).

\subsection{Additional analysis of human factors risks}

\subsubsection{Overview of previous analysis of risks}

A first identification of human factors related "issues" was developed during the analysis of functions in the earlier phase of work and this information was reformulated (also in the earlier phase of work [7], including much additional work with SMEs, to produce a human factors register. The register included more than 130 human factors related risks, along with information on the function in which the risk is possible and some preliminary details of causes (including use of TRACEr analyses for some of the risks [6]). Limited analyses were carried out for barriers or mitigations for specific risks. The specific risks have been classified into a number of "generic human factors" (listed in Table 3 ), a number of strategic themes that were implied within the larger list of risks. This was inspired by the Human Factors Case approach which has been developed at EUROCONTROL [2].

The identification of risks has so far focused on human factors that are likely to impact on safety risks, for project related reasons. Additional work will be necessary to extend the analysis to consider human factors risks that could result in production and operational issues.

Table 3 Summary of "generic human factors"

Planning, checks on documents
Identification of limits, locations, protection and layout of infra-
structure - (in relation to signals, points, junctions, and crossings)
(by signallers, track based staff)
Monitoring / checking / examination of occupancy or condition
of the infrastructure
Communications - agreement and understanding on possession /
protection limits and other protection arrangements
Instruction, supervision and control of workgroups
Instruction, supervision and control of movements
Response to situations / conditions
Response to situations / conditions

\subsubsection{Findings from the re-analysis of information on risks and groups of risks}

In the current review, information on the risks that are associated with a small sample of engineering activities has been collated and re-analysed. This has given an understanding of the range and depth of information that is available from analyses to date (e.g. in relation to the contexts in which activities take place, objectives of the activities, factors affecting performance of the activities and the types of problems that can occur). This re-analysis has looked at the specific risks which have been linked to an activity, the relevant generic human 
factors (groupings of risks), content on causes from the human factors register and outputs from TRACEr analyses (where available for specific risks) and likely outcomes of the human factors risks (using outputs from a mapping exercise to related safety analyses).

The small sample that has been considered included the following activities: (1) checks on standard documents, (2) establishing work areas and (3) coordination and safe working between groups.

Review of this small sample of activities shows the differences in the nature of the activities and types of information on risk that have been produced to date in analysis of human factors risks. For example, the first activity has been linked to one generic human factor (and only two specific risks), whereas the third activity has been associated with two generic human factors (with as many as 27 specific risks linked to one of these generic human factors). TRACEr analyses have been carried out for some, but not all, of the specific risks, giving a structured approach to the identification and description of errors (e.g. by cognitive domains, internal error modes and psychological error mechanisms) during an activity. To date, work has not been carried out to identify potential points of intervention (e.g. barriers, mitigation, other system related interventions to deal with interactions of factors).

These three examples give an indication of the depth of information available in outputs from analyses to date, though the effort in collating this for the analysis of these few examples has been considerable. More efficient ways of accessing this type of information are needed.

\section{Discussion and general conclusions from the re-analysis}

\subsection{What does the organisation know about roles, functions and descriptions of tasks relevant for engineering and maintenance work?}

Two stages of analysis which were inspired by phases of CWA have been used, providing a useful structure to organise a large amount of information that has been brought together from earlier pieces of work. At present, neither of the stages give sufficient depth of description of activity (e.g. see [1]). However, this preliminary analysis may be a stepping stone to additional analyses, such as more traditional CWA considering the constraints that shape work [4] or the modelling of properties in a system that shape the activity that will take place [5]. Nevertheless, the findings from this re-analysis are of practical value to Network Rail and the wider rail industry. For example, the organisation of the data could help with the dissemination of knowledge (e.g. within a knowledge management system or database). The structured outputs from this re-analysis are also useful in identifying where additional, detailed analysis are needed, to understand more about the functions, activities and roles in rail engineering, in a range of realistic situations, both now and in the future. Generally, a good understanding of the rail engineering system has been developed, but there is still more to be done. This is not a one off exercise and the understanding of the system will need to be refined as the system evolves. This work has provided the rudiments of a tool which can be used to consider the functions, activities and roles in the system as changes occur.

\subsection{What are the HF risks for today's (and unless things change), tomorrow's railway?}

A second part of the re-analysis has included the classification and interpretation of qualitative data on two types of information: the factors that can affect the performance of functions and the specific risks associated with these functions. The earlier, detailed work to understand functions of rail engineering has been an essential grounding for the work on identification of risks and a large number of specific human factors risks have been identified. The collation of risk related information for a selection of activities in the current review has highlighted the range of supporting information (e.g. on likely influencing factors) that has been generated in work so far. However, the effort required to assemble and interpret these outputs across a broader range of activities could be considerable without development of an appropriate database or knowledge based support system.

It is unlikely that all specific risks can be identified, due to differences in contexts and the potential interacting effects of components in the system. The current work has illustrated how there can be differences in the types and quantity of risks which have been linked to different activities. This may reflect the true nature of risk in these situations, or may be indicative of minor differences in the approach to risk identification, for practical reasons (e.g. when working with different SMEs). Never- 
theless, working at the level of specific risks has been a useful exercise and has been important to project staff in the process of developing rules for a new system.

\subsection{Determining organisational priorities for future work}

This review and re-analysis has provided the foundations for the development of a much smaller list of topics ("generic human factors") which, in conjunction with the classification of the factors affecting functions, represent a range of strategic issues that could be considered in future work. The generic human factors (Table 3 ) and the groupings of factors affecting functions (Table 2) seem to be complementary, through their focus on different aspect of the system. However, further work is needed to consider the degree of distinction or overlap between these sets of human factors (e.g. those impacting on the performance of functions or those that are groupings of risks). This may be indicative of different types of human factors in this rail engineering context. It might be possible in future work to identify characteristics of factors which influence different outcomes, such as successful performance, recovery, events, risks or the transition to more serious outcomes during incidents.

The likely value of working with groupings of factors (e.g. for factors affecting functions and for the grouping of risks into generic human factors) requires further consideration. In particular, it will be necessary to verify that the classifications are comprehensive and give a reliable indication of the main human factors risks in the system and the different facets of these risks. It will be important to establish how these groupings could be used at a strategic level in the organisation, through consultation with relevant stakeholders (e.g. could the "generic human factors" be developed for use as leading indicators, giving early indications of "loss of control" in the system?). In practice, it will be necessary to develop an approach which considers the right balance between the strategic issues (linked to the groupings of factors) and the tactical issues (linked to the specific risks and contextual findings).

Overall, the selected methods for representation of information on functions and activities which were obtained in earlier work, in conjunction with findings from additional qualitative analysis of descriptive data, have been useful in this complex, industrial context. The programme of work is on- going and there is more work that is needed, both in relation to the understanding of the functions and associated risks in the rail domain and for the development and application of the research methodology. Some of the next steps include:

- Validation of the outputs (in the draft hierarchical representation, the activity matrix and the classifications of strategic HF related issues) and future use of these to give a clearer picture of what is needed for collection of additional detail on some functions and associated risks.

- Specification of tools to support additional analysis and dissemination of the detailed, contextual information on the functions and risks in the system

- Consideration of more traditional use of CWA for analysis of relevant parts of the system (including decision ladders, social interactions, analyses of competencies)

- Development of an approach for in-depth analysis of barriers and mitigations for identified risks, with consideration given to control of system risks and interactions among system components [3]

- Extension of the work to identify additional production, operational and other risks associated with the system. This will need to be carried out in an efficient way, with consideration of a method of prioritising these different risks.

- Application of the methodology to the study of future systems.

\section{References}

[1] Daniellou, F. (2005) The French-Speaking Ergonomists' approach to work activity: Cross-influences of field intervention and conceptual models, Theoretical Issues in Ergonomics Science, 6, 405-427.

[2] Eurocontrol (2007) The Human Factors Case: Guidance for Human Factors Integration. Internal report from Eurocontrol.

[3] Leveson, N. (2011) Applying systems thinking to analyze and learn from events, Safety Science, 49, 55-64.

[4] Naikar, N., Moylan, A., Pearce, B. (2006) Analysing activity in complex systems with cognitive work analysis: concepts, guidelines and case study for control task analysis, Theoretical Issues in Ergonomics Science, 7, 371-394.

[5] Sanderson, P. (1999) Use of cognitive work analysis across the system life cycle: From requirements to decommissioning, Proceedings of the $43^{\text {rd }}$ Annual Meeting of the Human Factors and Ergonomics Society. 
[6] Shorrock, S. T. and Kirwan, B. (2002). Development and application of a human error identification tool for air traffic control, Applied Ergonomics, 33, 319-336.

[7] Wilson, J.R., Ryan, B., Schock, A, Ferreira, P., Smith, S., Pitsopoulos, J., (2009) Understanding risk in rail engineering work systems, Ergonomics, 52 (7), 774-790. 